H. MORI

KODAI MATH. J.

2 (1979), 243-253

\title{
REMARKS ON MEAN-VALUE INEQUALITIES ON $m$-DIMENSIONAL SUBMANIFOLDS OF $S^{n}$
}

Dedicated to Professor Tominosuke Ōtsuki on his 60th birthday

\author{
By HiROSHI MORI
}

1. Introdcution. In a work [5], J. Michael and L. Simon studied meanvalue inequalities on generalized submanifolds of $R^{n}$, and from which the volume of domains in an $m$-dimensional submanifold of $R^{n}$ can be estimated under additional assumptions (see also [6]). In this note, we study mean-value inequalities for submanifolds of the $n$-dimensional unit sphere $S^{n}$ and then we estimate the volume of domains in an $m$-dimensional submanifold $M$ of $S^{n}$ under certain additional assumptions.

2. Statement of results. Let $M$ be a $m$-dimensional immersed submanifold of $S^{n}$. We use the following quantities:

$H=$ the mean curvature vector field of $M$,

$\omega_{m}=$ the volume of the unit ball in $R^{m}$,

$B_{\rho}(\xi)=$ the open geodesic ball in $S^{n}$ with center $\xi$ and radius $\rho$,

$S_{\rho}(\xi)=M \cap B_{\rho}(\xi)$.

2.1 THEOREM (Mean-value Inequality). Let $\chi$ be a non-negative (weakly) subharmonic function on $M$ and suppose that there is a constant $\Lambda$ such that $|H(x)| \leqq \Lambda$ for all $x \in M$. For each $\xi \in M$ and for each $\rho \in\left(0, \frac{\pi}{2}\right)$, the inequality

$$
\omega_{m}(\sin \rho)^{m} \chi(\xi) \leqq h(\rho) \int_{S_{\rho}(\xi)} \chi(x) d V(x)
$$

holds, where $d V$ is the volume element of $M$ and

$$
\begin{aligned}
h(\rho)= & \min \cdot\left\{\exp \Lambda \rho, 1+\Lambda \rho+\frac{\left(\frac{\pi}{2}\right)^{1+1}}{2 !} \Lambda^{2} \rho \sin \rho+\cdots\right. \\
& \left.+\frac{\left(\frac{\pi}{2}\right)^{1+2+\cdot \cdot(m-1)+(m-1)}}{m !} \Lambda^{m} \rho \sin ^{m-1} \rho\right\} .
\end{aligned}
$$

Received January 24, 1978. 
Theorem 2.1 has immediate geometric applications. By putting $\chi \equiv 1$, we have

2.2 Corollary. If $M$ has no boundary in $S_{\rho}(\xi)$ for some $\xi \in M$ and for some $\rho \in\left(0, \frac{\pi}{2}\right)$, then

$$
\text { volume } S_{\rho}(\xi) \geqq \omega_{m}(\sin \rho)^{m} / h(\rho) .
$$

2.3 COROLLARY. If furthermore $M$ is minmally zmmersed, then

$$
\text { volume } S_{\rho}(\xi) \geqq \omega_{m}(\sin \rho)^{m} \text {. }
$$

The author would like to thank Professor H. Kitahara for his kind advice and encouragement.

3. Preliminaries. We denote by $\langle$, $\rangle$ the canonical Riemannian metric on $S^{n}$ induced from $R^{n+1}$ and by $\bar{\nabla}$ the canonical Riemannian connection on $S^{n}$. Let $M$ be a $C^{\infty} m$-dimensional immersed submanifold of $S^{n}$. We denote by $\langle$,$\rangle and \nabla$ the induced Riemannian metric and the Riemannian connection on $M$ respectively. We denote the tangent and normal bundles of $M$ by $T M$ and $N M$ respectively, and denote the projection of a vector field $X$ along $M$ into $T M, N M$ by $X^{T}, X^{N}$ respectively. The second fundamental form $B: T_{x}(M) \times$ $T_{x}(M) \rightarrow N_{x}(M)$ at $x$ is given by

$$
B(X, Y)=\bar{\nabla}_{X} \bar{Y}-\left(\bar{\nabla}_{X} \bar{Y}\right)^{T}=\bar{\nabla}_{X} \bar{Y}-\nabla_{X} \bar{Y}, \quad X, Y \in T_{x}(M),
$$

where $\bar{Y}$ is a smooth extension of $Y, T_{x}(M)$ is the tangent space to $M$ at $x$ and $N_{x}(M)$ is the normal space to $T_{x}(M)$ in $T_{x}\left(S^{n}\right)$. The mean curvature vector field $H$ of $M$ is given by $H=$ trace of $B$. We denote by $\Delta$ the Laplace operator acting on $C^{2}(M)$, the space of class $C^{2}$ functions on $M$. A real valued function $\chi$ on $M$ will be called (weakly) subharmonic if $\chi$ is continuous on $M$ and

$$
\int_{M} \chi(x) \Delta h(x) d V(x) \geqq 0
$$

for every non-negative function $h \in C_{0}^{2}(M)$, the space of class $C^{2}$ functions on $M$ with compact support.

It can be easily shown that for $g \in C_{0}^{2}(M)$ and $h \in C^{2}(M)$

$$
\int_{M} \Delta g \cdot h d V=-\int_{M}\langle\nabla g, \nabla h\rangle d V
$$

and so that

$$
\int_{M} \Delta g \cdot h d V=\int_{M} g \cdot \Delta h d V
$$

where $\nabla g$ is the gradient vector field of $g$. Therefore, it follows that $\chi \in C^{2}(M)$ 
is (weakly) subharmonic if and only if $\Delta \chi \geqq 0$ on $M$. For a fixed point $\xi \in M$ we denote the geodesic distance in $S^{n}$ from $\xi$ to $x \in S^{n}$ by $r(x)$. For later applications we prove the following Lemma.

3.1 Lemia. For each point $x \in M$ with $0<r(x)<\pi$ we have

$$
(\Delta r)(x)=m \cot r(x)+\langle\gamma(r(x)), H(x)\rangle-\langle e, \dot{\gamma}(r(x))\rangle^{2} \cot r(x),
$$

where $\gamma$ is the unque minmal geodesic with unt speed in $S^{n}$ from $\xi$ to $x$ and $e$ is the vector in $T_{x}(M)$ with $|e|=1$ such that $\langle e, \dot{\gamma}(r(x))\rangle=\max .\{\langle X, \dot{\gamma}(r(x))\rangle ; X \in$ $\left.T_{x}(M),|X|=1\right\}$.

Proof. For any point $x \in M$ and any $X \in T_{x}(M)$, let $c:(-\varepsilon, \varepsilon) \rightarrow M$ for some $\varepsilon>0$ be the geodesic such that $c(0)=x$ and $\dot{c}(0)=X$. If $0<r(x)<\pi$, then we can choose a sufficiently small $\varepsilon>0$ such that $r(c(t))=\operatorname{Cos}^{-1}(\langle\xi, c(t)\rangle), t \in$ $(-\varepsilon, \varepsilon)$, where $\operatorname{Cos}^{-1} u$ is the principal value of arc cosine $u, 0 \leqq u \leqq 1$. Then we have

$$
\begin{aligned}
(X \cdot r)(x) & =\left.\frac{d}{d t} r(c(t))\right|_{t=0} \\
& =\left.\frac{-\langle\xi, \dot{c}(t)\rangle}{\sin \left[\operatorname{Cos}^{-1}(\langle\xi, c(t)\rangle)\right]}\right|_{t=0} \\
& =\frac{-\langle\xi, X\rangle}{\sin r(x)} .
\end{aligned}
$$

Furthermore, extending $\dot{c}(t)$ as a vector field around $x$ and denoting it by the same notation $X$ we have

$$
\begin{aligned}
(X(X \cdot r))= & \left.\frac{d}{d t} \frac{-\langle\xi, \dot{c}(t)\rangle}{\sin \left[\operatorname{Cos}^{-1}(\langle\xi, c(t)\rangle)\right]}\right|_{t=0} \\
= & \left.\frac{-\langle\xi, \ddot{c}(t)\rangle}{\sin \left[\operatorname{Cos}^{-1}(\langle\xi, c(t)\rangle)\right]}\right|_{t=0} \\
& +\frac{\langle\xi, \dot{c}(t)\rangle}{\sin ^{2}\left[\operatorname{Cos}^{-1}(\langle\xi, c(t)\rangle)\right]} \times\left.\frac{d}{d t} \sin \left[\operatorname{Cos}^{-1}\langle\xi, c(t)\rangle\right]\right|_{t=0} \\
= & \frac{-\langle\xi, \ddot{c}(o)\rangle}{\sin r(x)}-\frac{\cos r(x)}{\sin ^{3} r(x)}\langle\xi, X\rangle^{2} .
\end{aligned}
$$

Let $\left\{e_{j}\right\}$ be an orthonormal basis of $T_{x}(M)$ such that $e_{1}=e$, and let each $e_{j}$ extend to $E$, around $x$ such that $E_{\jmath}$ is the tangent vector along the geodesic emanating from $x$ with the tangent vector $e_{\jmath}$. Then from the above argument we have

$$
(\Delta r)(x):=\sum_{j=1}^{m}\left\{\left(\nabla_{E_{j}}\left(\nabla_{E_{j}} r\right)\right)(x)-\left(\left(\nabla_{E_{j}} E_{\jmath}\right) r\right)(x)\right\}
$$




$$
\begin{aligned}
& =\sum_{\jmath=1}^{m}\left\{E_{\jmath}\left(E_{j} r\right)\right\}(x) \\
& =\frac{-1}{\sin r(x)}\left\langle\xi, \sum_{\jmath=1}^{m} \tilde{\nabla}_{E_{j}} E_{\jmath}\right\rangle(x)-\frac{\cos r(x)}{\sin ^{3} r(x)} \sum_{\jmath=1}^{m}\left\langle\xi, e_{\jmath}\right\rangle^{2}(x),
\end{aligned}
$$

where $\tilde{\nabla}$ is the canonical connection of $R^{n+1}$.

On the other hand, from the formulas of Gauss we have

$$
\begin{aligned}
\left(\tilde{\nabla}_{E_{j}} E_{\jmath}\right)(x) & =\left(\bar{\nabla}_{E_{\jmath}} E_{\jmath}(x)-\left\langle E_{\jmath}, E_{\jmath}\right\rangle(x) x\right. \\
& =\left(\nabla_{E_{j}} E_{j}\right)(x)+B\left(E_{\jmath}, E_{\jmath}\right)(x)-\left\langle e_{\jmath}, e_{\jmath}\right\rangle x \\
& =B\left(e_{\jmath}, e_{j}\right)-x,
\end{aligned}
$$

while, from the choice of $\left\{e_{j}\right\}$ we have

$$
\left\langle e_{\jmath}, \xi\right\rangle=0 \quad \text { for } \quad \jmath=2,3, \cdots, m \text {, }
$$

and

$$
\left\langle e_{1}, \xi\right\rangle=\langle e, \xi\rangle \text {. }
$$

And since $r(x)$ satisfies $0<r(x)<\pi$, it is easily shown that $\dot{\gamma}(r(x))$ is represented as an element of $R^{n+1}$

$$
\dot{\gamma}(r(x))=-\frac{-1}{\sin r(x)} \xi+\cot r(x) x .
$$

From (1), (2), (3), (4) and (5) we get

$$
\begin{aligned}
(\Delta r)(x)= & \frac{-1}{\sin r(x)}\left\langle\xi, \sum_{j=1}^{m} B\left(e_{\jmath}, e_{j}\right)\right\rangle+\frac{1}{\sin r(x)}\langle\xi, m x\rangle \\
& -\frac{\cos r(x)}{\sin ^{3} r(x)}\langle e, \xi\rangle^{2} \\
= & m \cot r(x)+\langle\dot{\gamma}(r(x)), H(x)\rangle-\langle e, \dot{\gamma}(r(x))\rangle^{2} \cot r(x)
\end{aligned}
$$

by virtue of $H(x):=\sum_{j=1}^{m} B\left(e_{j}, e_{j}\right)$ and $\langle\xi, x\rangle=\cos r(x)$. This completes the proof.

4. Proof of the mean-value inequality. Let $\lambda$ be a nondecreasing $C^{1}$ function on $R$ with $\lambda(t)=0$ for $t \leqq 0$. Let $\chi$ be a non-negative (weakly) subharmonic function on $M$. For a fixed point $\xi \in M$ we define the following quantities:

$$
\begin{aligned}
& \phi_{\xi}(\rho)=\int_{M} \chi(x) \lambda(\rho-r(x)) d V(x), \\
& \phi_{\xi}(\rho)=\int_{M} \chi(x)|H(x)| \lambda(\rho-r(x)) d V(x) .
\end{aligned}
$$


We now prove the following Lemma.

4.1 Lemma. Let $\phi_{\xi}(\rho)$ and $\phi_{\xi}(\rho)$ be as above. Then

$$
-\frac{d}{d \rho}\left(\frac{\phi_{\xi}(\rho)}{\sin ^{m} \rho}\right) \leqq \frac{1}{\rho \sin ^{m} \rho} \int_{0}^{\rho} \sigma \psi_{\xi}^{\prime}(\sigma) d \sigma
$$

for each $\rho \in\left(0, \frac{\pi}{2}\right)$.

Proof. Let $f$ be defined on $R$ by

$$
f(s)=\int_{s}^{\infty} t \lambda(\rho-t) d t .
$$

Then for any $\rho \in(0, \pi)$ the support of the function for, $r=r(x)$, is contained in the closure of the set $B_{\rho}(\xi)$ and $f \circ r$ is $C^{2}$ function on $S^{n}-\{\xi\}$ by virtue of $r=r(x)$ is $C^{\infty}$ function on $B_{\rho}(\xi)-\{\xi\}$. We now show that $f \circ r$ can be considered as a $C^{2}$ function at $\xi$. Since the exponential map $\exp _{\xi}$ of $S^{n}$ at $\xi$ induces the diffeomorphism of the set $\left\{X \in T_{\xi}\left(S^{n}\right) ;|X|<\pi\right\}$ onto the set $\left\{x \in S^{n} ; r(x)<\pi\right\}$ it is sufficient to show that $f \circ r \circ \exp _{\xi}$ can be considered as a $C^{2}$ function at the origin of $T_{\hat{\xi}}\left(S^{n}\right)$. Let $\left\{x^{i}\right\}$ be the coordinate system of $T_{\xi}\left(S^{n}\right)$ with respect to some orthonormal basis of $T_{\xi}\left(S^{n}\right)$. Since $\left(r \circ \exp _{\xi}\right)\left(x^{1}, \cdots, x^{n}\right)=\left(\sum_{k=1}^{n}\left(x^{k}\right)^{2}\right)^{1 / 2}$ we can show that for $\left(x^{1}, \cdots, x^{n}\right) \neq(0, \cdots, 0)$

$$
\begin{aligned}
& \left.\frac{\partial}{\partial x^{2}}\left(f \circ r \circ \exp _{\xi}\right)\left(x^{1}, \cdots, x^{n}\right)=-\lambda\left[\rho-\left(\sum_{k=1}^{n}\left(x^{k}\right)^{2}\right)^{1 / 2}\right)\right] x^{2}, \\
& \frac{\partial^{2}}{\partial x^{\jmath} \partial x^{2}}\left(f \circ r \circ \exp _{\xi}\right)\left(x^{1}, \cdots, x^{n}\right)=-\lambda\left[\rho-\left(\sum_{k=1}^{n}\left(x^{k}\right)^{2}\right)^{1 / 2}\right] \delta_{\imath \jmath} \\
& +\lambda^{\prime}\left[\rho-\left(\sum_{k=1}^{n}\left(x^{k}\right)^{2}\right)^{1 / 2}\right] \frac{x^{2} x^{\jmath}}{\left(\sum_{k=1}^{n}\left(x^{k}\right)^{2}\right)^{1 / 2}}
\end{aligned}
$$

From this we see that $f \circ r \circ \exp _{\xi}$ can be extended as a $C^{2}$ function around $\xi$. For each point $x \in M$ with $0<r(x)<\pi$ let $\left\{e_{\}}\right\}$be an orthonormal basis of $T_{x}(M)$ as in the proof of Lemma 3.1. Then we have

$$
\begin{aligned}
\Delta(f \supset r)(x)=-r(x) \lambda(\rho-r(x)) \Delta r(x)- & {\left[\lambda(\rho-r(x))-r(x) \lambda^{\prime}(\rho-r(x))\right] } \\
& \times|\nabla r(x)|^{2},
\end{aligned}
$$

where $|\nabla r(x)|^{2}=\sum_{j=1}^{m}\left[\left(e_{j} r\right)(x)\right]^{2}$.

Using the unique minimal geodesic $\gamma$ in $S^{n}$ from $\xi$ to $x$, and from the choice of $\left\{e_{j}\right\}$ we have

$$
|\nabla r(x)|^{2}=\frac{\langle\xi, e\rangle^{2}}{\sin ^{2} r(x)}=\langle e, \dot{\gamma}(r(x))\rangle^{2} .
$$

From Lemma 3.1 we get 
(6)

$$
\begin{aligned}
\Delta(f \circ r)(x)= & -m r(x) \cot r(x) \cdot \lambda(\rho-r(x)) \\
& -r(x) \lambda(\rho-r(x))\langle\dot{\gamma}(r(x)), H(x)\rangle \\
& +r(x) \cot r(x) \cdot \lambda(\rho-r(x))\langle e, \dot{\gamma}(r(x))\rangle^{2} \\
& -\left\{\lambda(\rho-r(x))-r(x) \lambda^{\prime}(\rho-r(x))\right\}\langle e, \dot{\gamma}(r(x))\rangle^{2} .
\end{aligned}
$$

Since $0<u \cot u<1$ for $u$ in $\left(0, \frac{\pi}{2}\right)$ we get

$$
\begin{gathered}
r(x) \cot r(x) \lambda(\rho-r(x))\langle e, \dot{\gamma}(r(x))\rangle^{2} \\
-\lambda(\rho-r(x))\langle e, \dot{\gamma}(r(x))\rangle^{2} \leqq 0 .
\end{gathered}
$$

From (6) and (7) we obtain the following inequality

$$
\begin{aligned}
\Delta(f \circ r)(x) \leqq & -m r(x) \cot r(x) \cdot \lambda(\rho-r(x)) \\
& +r(x) \lambda(\rho-r(x))|H(x)|+r(x) \lambda^{\prime}(\rho-r(x))
\end{aligned}
$$

so that we have by the properties of $\chi$ and $f \circ r$

$$
\begin{aligned}
0 \leqq & \int_{M} \chi(x) \Delta(f \circ r)(x) d V(x) \\
\leqq & -m \int_{M} r(x) \cot r(x) \cdot \chi(x) \lambda(\rho-r(x)) d V(x) \\
& +\int_{M} \chi(x) r(x) \lambda(\rho-r(x))|H(x)| d V(x) \\
& +\int_{M} \chi(x) r(x) \lambda^{\prime}(\rho-r(x)) d V(x)
\end{aligned}
$$

for each $\rho \in\left(0, \frac{\pi}{2}\right)$.

From the inequalities $r \lambda^{\prime}(\rho-r) \leqq \rho \lambda^{\prime}(\rho-r)$ and $r \cot r \geqq \rho \cot \rho$ for $0<r \leqq$ $\rho\left(\leqq \frac{\pi}{2}\right)$ we get

and

$$
\begin{aligned}
& -m \int_{M} r(x) \cot r(x) \cdot \chi(x) \lambda(\rho-r(x)) d V(x) \\
& \leqq-m \rho \cot \rho \cdot \phi_{\xi}(\rho)
\end{aligned}
$$

$$
\int_{M} \chi(x) r(x) \lambda^{\prime}(\rho-r(x)) d V(x) \leqq \rho \phi_{\xi}^{\prime}(\rho)
$$

while 


$$
\begin{aligned}
& \int_{M} \chi(x)|H(x)| r(x) \lambda(\rho-r(x)) d V(x) \\
& \quad=\int_{M} \chi(x)|H(x)|\left\{\int_{0}^{\rho} r(x) \lambda^{\prime}(\sigma-r(x)) d \sigma\right\} d V(x) \\
& \quad \leqq \int_{M} \chi(x)|H(x)|\left\{\int_{0}^{\rho} \sigma \lambda^{\prime}(\sigma-r(x)) d \sigma\right\} d V(x) \\
& =\int_{0}^{\rho} \sigma \psi_{\xi}^{\prime}(\sigma) d \sigma .
\end{aligned}
$$

Hence (8) gives

$$
m \rho \cot \rho \phi_{\xi}(\rho)-\rho \phi_{\xi}^{\prime}(\rho) \leqq \int_{0}^{\rho} \sigma \phi_{\xi}^{\prime}(\sigma) d \sigma,
$$

which can be written in the form

$$
-\frac{d}{d \rho}\left(\frac{\phi_{\xi}(\rho)}{\sin ^{m} \rho}\right) \leqq \frac{1}{\rho \sin ^{m} \rho} \int_{0}^{\rho} \sigma \phi_{\xi}^{\prime}(\sigma) d \sigma
$$

as required.

Noting that

$$
\int_{0}^{\rho} \sigma \psi_{\xi}^{\prime}(\sigma) d \sigma \leqq \rho \int_{0}^{\rho} \psi_{\xi}^{\prime}(\sigma) d \sigma=\rho \psi_{\xi}(\rho)
$$

we obtain the following Corollary to Lemma 4.1.

4.2 COROLlARY. If the hypotheses are as in Lemma 4.1, then

$$
-\frac{d}{d \rho}\left(\frac{\phi_{\xi}(\rho)}{\sin ^{m} \rho}\right) \leqq \frac{\phi_{\xi}(\rho)}{\sin ^{m} \rho}
$$

for each $\rho \in\left(0, \frac{\pi}{2}\right)$.

Proof of Theorem 2.1. We follow essentially the argument of [5]. We first show the following inequality

$$
\begin{gathered}
\omega_{m}(\sin \rho)^{m} \chi(\xi) \leqq\{1+\Lambda \rho+ \\
+\frac{\left(\frac{\pi}{2}\right)^{1+1}}{2 !} \Lambda^{2} \rho \sin \rho+\cdots+\frac{\left(\frac{\pi}{2}\right)^{1+2+\cdots+(m-1)+(m-1)}}{m !} \\
\left.\times \Lambda^{m} \rho \sin ^{m-1} \rho\right\} \int_{S_{\xi}(\rho)} \chi(x) d V(x) .
\end{gathered}
$$

Let $\phi_{\xi}, \phi_{\xi}$ be as in Lemma 4.1. Since $|H(x)| \leqq \Lambda$ for all $x \in M$, we have $\phi_{\xi}^{\prime}(\rho) \leqq \Lambda \phi_{\xi}^{\prime}(\rho)$ and hence Lemma 4.1 implies

$$
-\frac{d}{d \rho}\left(\frac{\phi_{\xi}(\rho)}{\sin ^{m} \rho}\right) \leqq \frac{\Lambda}{\rho \sin ^{m} \rho} \int_{0}^{\rho} \sigma \dot{\phi}_{\xi}^{\prime}(\sigma) d \sigma
$$


for each $\rho \in\left(0, \frac{\pi}{2}\right)$. Let $\rho \in\left(0, \frac{\pi}{2}\right)$ be fixed.

Using the notation

$$
\begin{aligned}
T_{j} & =\int_{0}^{\rho} \frac{1}{\sigma \sin ^{j} \sigma}\left(\int_{0}^{\sigma} \tau \phi_{\xi}^{\prime}(\tau) d \tau\right) d \sigma \\
& =\lim _{\varepsilon \rightarrow 0} \int_{\varepsilon}^{\rho} \frac{1}{\sigma \sin ^{j} \sigma}\left(\int_{0}^{\sigma} \tau \phi_{\xi}^{\prime}(\tau) d \tau\right) d \sigma
\end{aligned}
$$

for $0 \leqq \jmath \leqq m$ and integrating (10) over the interval $(\sigma, \rho)$ (where $0<\sigma<\rho$ ), we first obtain

$$
\frac{\phi_{\xi}(\sigma)}{\sin ^{m} \sigma} \leqq \frac{\phi_{\xi}(\rho)}{\sin ^{m} \rho}+\Lambda T_{m}
$$

Next, using integration by parts, we get

$$
\begin{aligned}
T_{J} & =\lim _{\varepsilon \rightarrow 0}\left\{\left.\left(\int_{\rho}^{\sigma} \frac{d \tau}{\tau \sin ^{\jmath} \tau}\right)\left(\int_{0}^{\sigma} \tau \phi_{\xi}^{\prime}(\tau) d \tau\right)\right|_{\sigma=\varepsilon} ^{\sigma=\rho}-\int_{\varepsilon}^{\rho}\left(\int_{\rho}^{\sigma} \frac{d \tau}{\tau \sin ^{j} \tau}\right) \sigma \phi_{\xi}^{\prime}(\sigma) d \sigma\right\} \\
& =\lim _{\varepsilon \rightarrow 0} \int_{\varepsilon}^{\rho} \sigma\left(\int_{\sigma}^{\rho} \frac{d \tau}{\tau \sin ^{j} \tau}\right) \phi_{\xi}^{\prime}(\sigma) d \sigma \\
& =\lim _{\varepsilon \rightarrow 0}\left\{\left.\sigma\left(\int_{\sigma}^{\rho} \frac{d \tau}{\tau \sin ^{j} \tau}\right) \phi_{\xi}(\sigma)\right|_{\sigma=\varepsilon} ^{\sigma=\rho}\right. \\
& \left.-\int_{\varepsilon}^{\rho} \phi_{\xi}(\sigma)\left(\int_{\sigma}^{\rho} \frac{d \tau}{\tau \sin ^{j} \tau}-\frac{1}{\sin ^{\jmath} \sigma}\right) d \sigma\right\} \\
& =\int_{0}^{\rho} \frac{\phi_{\xi}(\sigma)}{\sin ^{\jmath} \sigma} d \sigma-\int_{0}^{\rho} \phi_{\xi}(\sigma)\left(\int_{\sigma}^{\rho} \frac{d \tau}{\tau \sin ^{\jmath} \tau}\right) d \sigma .
\end{aligned}
$$

Since $\frac{1}{\tau \sin ^{j} \tau} \geqq \frac{\cos \tau}{\sin ^{j+1} \tau}$ for $0<\tau<\frac{\pi}{2}$, it follows that

$$
\begin{gathered}
-\int_{0}^{\rho} \phi_{\xi}(\sigma)\left(\int_{\sigma}^{\rho} \frac{d \tau}{\tau \sin ^{\jmath} \tau}\right) d \sigma \leqq \int_{0}^{\rho} \phi_{\xi}(\sigma) \frac{1}{\jmath}\left(\frac{1}{\sin ^{\jmath} \rho}-\frac{1}{\sin ^{\jmath} \sigma}\right) d \sigma \\
\leqq \frac{1}{\jmath} \frac{\rho}{\sin ^{\jmath} \rho} \phi_{\xi}(\rho)-\frac{1}{\jmath} \int_{0}^{\rho} \frac{\phi_{\xi}(\sigma)}{\sin ^{\jmath} \sigma} d \sigma .
\end{gathered}
$$

Now we can write

$$
\int_{0}^{\rho} \frac{\phi_{\xi}(\sigma)}{\sin ^{\jmath} \sigma} d \sigma=\int_{0}^{\rho} \frac{\phi_{\xi}(\sigma)}{\sin ^{m} \sigma} \sin ^{m-\jmath} \sigma d \sigma,
$$

so that by integrating by parts and using (10) and the inequalities

$$
\int_{0}^{\sigma} \sin ^{m-\jmath} \tau d \tau \leqq \frac{\sigma^{m-\jmath+1}}{m-\jmath+1} \text { and } \sigma^{m-\jmath+1} \leqq\left(\frac{\pi}{2} \sin \sigma\right)^{m-j+1} \text { for } 0<\sigma<\frac{\pi}{2},
$$

we obtain 


$$
\begin{aligned}
& \int_{0}^{\rho} \frac{\phi_{\xi}(\sigma)}{\sin ^{\jmath} \sigma} d \sigma=\lim _{\varepsilon \rightarrow 0} \int_{\varepsilon}^{\rho} \frac{\phi_{\xi}(\sigma)}{\sin ^{m} \sigma} \sin ^{m-\jmath} \sigma d \sigma \\
& =\lim _{\varepsilon \rightarrow 0}\left\{\left.\frac{\phi_{\xi}(\sigma)}{\sin ^{m} \sigma} \int_{0}^{\sigma} \sin ^{m-\jmath} \tau d \tau\right|_{\sigma=\varepsilon} ^{\sigma=\rho}\right. \\
& \left.+\int_{\varepsilon}^{\rho}-\frac{d}{d \sigma}\left(\frac{\phi_{\xi}(\sigma)}{\sin ^{m} \sigma}\right) \cdot\left(\int_{0}^{\sigma} \sin ^{m-\jmath} \tau d \tau\right) d \sigma\right\} \\
& \leqq \lim _{\varepsilon \rightarrow 0}\left\{\left.\frac{\phi_{\xi}(\sigma)}{\sin ^{m} \sigma} \int_{0}^{\sigma} \sin ^{m-\jmath} \tau d \tau\right|_{\sigma=\varepsilon} ^{\sigma=\rho}\right. \\
& \left.+\int_{\varepsilon}^{\rho} \frac{\Lambda}{\sigma \sin ^{m} \sigma}\left(\int_{0}^{\sigma} \tau \phi_{\xi}^{\prime}(\tau) d \tau\right)\left(\int_{0}^{\sigma} \sin ^{m-\jmath} \tau d \tau\right) d \sigma\right\} \\
& \leqq \frac{\phi_{\xi}(\rho)}{\sin ^{m} \rho} \int_{0}^{\rho} \sin ^{m-\jmath} \tau d \tau+\int_{0}^{\rho} \frac{\Lambda}{\sigma \sin ^{m} \sigma} \frac{\sigma^{m-\jmath+1}}{m-j+1}\left(\int_{0}^{\sigma} \tau \phi_{\xi}^{\prime}(\tau) d \tau\right) d \sigma \\
& \leqq \frac{\phi_{\xi}(\rho)}{\sin ^{m} \rho} \frac{\rho^{m-\jmath+1}}{m-\jmath+1}+\int_{0}^{\rho} \frac{\Lambda}{m-\jmath+1} \frac{\left(\frac{\pi}{2} \sin \sigma\right)^{m-\jmath+1}}{\sigma \sin ^{m} \sigma}\left(\int_{0}^{\sigma} \tau \phi_{\xi}^{\prime}(\tau) d \tau\right) d \sigma \\
& =\frac{\rho^{m-\jmath+1}}{m-\jmath+1} \frac{\phi_{\xi}(\rho)}{\sin ^{m} \rho}+\Lambda \frac{\left(\frac{\pi}{2}\right)^{m-\jmath+1}}{m-\jmath+1} T_{\jmath-1} .
\end{aligned}
$$

Combining (12), (13) and (14) we have

$$
\begin{aligned}
T_{\jmath} \leqq & \frac{\jmath-1}{\jmath}\left\{\frac{\rho \phi_{\xi}(\rho)}{\sin ^{\jmath} \rho} \frac{1}{m-\jmath+1}\left(\frac{\rho}{\sin \rho}\right)^{m-\jmath}+\frac{\Lambda\left(\frac{\pi}{2}\right)^{m-\jmath+1}}{m-\jmath+1} T_{\jmath-1}\right\} \\
& \quad+\frac{1}{\jmath} \frac{\rho \phi_{\xi}(\rho)}{\sin ^{\jmath} \rho} \\
\leqq & \frac{m\left(\frac{\pi}{2}\right)^{m-\jmath}}{j(m-\jmath+1)} \frac{\rho}{\sin ^{\jmath} \rho} \phi_{\xi}(\rho)+\frac{(\jmath-1)\left(\frac{\pi}{2}\right)^{m-\jmath+1}}{j(m-\jmath+1)} \Lambda T_{\jmath-1},
\end{aligned}
$$

for $1 \leqq \jmath \leqq m$. Inequality (15) for $\jmath=1,2, \cdots, m$ together with (10) implies

$$
\begin{gathered}
\frac{\phi_{\xi}(\sigma)}{\sin ^{m} \sigma} \leqq\left\{1+\frac{\Lambda \rho}{1 !}+\frac{\left(\frac{\pi}{2}\right)^{1+1}}{2 !} \Lambda^{2} \rho \sin \rho+\cdots+\frac{\left(\frac{\pi}{2}\right)^{1+2+\cdots+(m-1)+(m-1)}}{m !}\right. \\
\left.\times \Lambda^{m} \rho \sin ^{m-1} \rho\right\} \frac{\phi_{\xi}(\rho)}{\sin ^{m} \rho} .
\end{gathered}
$$

Now let $\varepsilon \in(0, \sigma)$ and choose the function $\lambda$ such that $\lambda(t) \geqq 1$ when $t \geqq \varepsilon$. Then (16) implies 


$$
\begin{aligned}
& \frac{1}{\sin ^{m} \sigma} \int_{S_{\sigma-\xi(\xi)}} \chi d V \leqq\left\{1+\frac{\Lambda \rho}{1 !}+\frac{\left(\frac{\pi}{2}\right)^{1+1}}{2 !} \Lambda^{2} \rho \sin \rho+\cdots+\right. \\
& \left.+\frac{\left(\frac{\pi}{2}\right)^{1+2+\cdots+(m-1)+(m-1)}}{m !} \Lambda^{m} \rho \sin ^{m-1} \rho\right\} \frac{1}{\sin ^{m} \rho} \int_{S_{\rho}(\xi)} \chi d V .
\end{aligned}
$$

Since $\sigma \in(0, \rho)$ and $\varepsilon \in(0, \sigma)$ are arbitrary we see that (9) is true.

Next we show the following inequality

$$
\omega_{m} \sin ^{m} \rho \chi(\xi) \leqq(\exp \Lambda \rho) \int_{S_{\rho}(\xi)} \chi(x) d V(x) .
$$

From the assumption on the mean curvature vector field $H$ and Corollary 4.2 we have

$$
-\frac{d}{d \sigma}\left(-\frac{\phi_{\xi}(\sigma)}{\sin ^{m} \sigma}\right) \leqq \Lambda \frac{\phi_{\xi}(\sigma)}{\sin ^{m} \sigma}
$$

for each $\sigma \in\left(0, \frac{\pi}{2}\right)$. Let $\rho \in\left(0, \frac{\pi}{2}\right)$ be fixed. Integrating (18) over the interval $(\sigma, \rho)$ (where $0<\sigma<\rho)$, we obtain

$$
\frac{\phi_{\xi}(\sigma)}{\sin ^{m} \sigma} \leqq(\exp \Lambda \rho) \frac{\phi_{\xi}(\rho)}{\sin ^{m} \rho}
$$

By virtue of $\frac{\phi_{\xi}(\sigma)}{\sin ^{m} \sigma} \geqq 0$ for $0<\sigma<\frac{\pi}{2}$.

Using (19) and repeating the same discussion as in the proof of (9), we obtain the inequality (17).

From (9) and (17) we see that our assertion is true.

Remark 1. Let $M$ be an $m$-dimensional minimal submanifold in $S^{n}$ which has no boundary in $S_{\rho}(\xi)=B_{\rho}(\xi) \cap M$ for some $\xi \in M$ and for some $\rho$ in $\left(\frac{\pi}{2}, \pi\right)$. Since volume $S_{\rho}(\xi)$ is a continuous function of $\xi, 0<\rho<\pi$, we have volume $S_{\pi / 2}(\xi) \geqq \omega_{m}$ by virtue of Corollary 2.3. This shows that our estimation of the volume of an $m$-dimensional compact minimal submanifold $M$ in $S^{n}$ with $r(x)$ $>\pi / 2$ for some point $x \in M$ will be better than the one of Hoffman and Spruck's (see [2]). We notice that the Clifford minimal hypersurfaces $M=S^{p}(\sqrt{p / m}) \times$ $S_{q}(\sqrt{q / m})$ in $S^{n}, p, q \geqq 1, p+q=m, n=m+1$, satisfy the conditions mentioned above.

Remark 2. Let $M$ be as in Corollary 2.3. When we consider $M$ as an $m$ dimensional submanifold of $R^{n+1}$ the norm of its mean curvature vector field is just $m$. Applying the Theorem 3.4 in [5] to this case we get

volume $M \cap U_{2 \sin \rho / 2}(\xi) \geqq \omega_{m}\left(2 \sin \frac{\rho}{2}\right)^{m} /\left\{1+2 m \sin \frac{\rho}{2}+\cdots+\left(2 m \sin \frac{\rho}{2}\right)^{m} / m !\right\}$. 
In this case, for $\rho$ sufficiently near $\pi / 2$ our estimation of the volume of such a domain in $M$ is better than the one of Michael and Simon's. Here $U_{\sigma}(\xi)$ is the open ball in $R^{n+1}$ with center $\xi$ and radius $\sigma$. We notice that $S^{n} \cap U_{2 \sin \sigma / 2}(\xi)$ $=B_{\sigma}(\xi)$ for $\xi \in S^{n}$ and $\sigma$ in $(0, \pi)$.

Remark 3. In the Theorem 2.1 we have

$$
\exp \Lambda \rho<f_{m}(\rho):=1+\Lambda \rho+\cdots+\frac{\left(\frac{\pi}{2}\right)^{1+2+\cdots+(m-1)+(m-1)}}{m !} \Lambda^{m} \rho \sin ^{m-1} \rho
$$

for sufficiently small $\rho>0$, provided $\Lambda \neq 0$. But it is not always true that exp $\Lambda \rho<f_{m}(\rho)$ for $\rho, 0<\rho<\pi / 2, \Lambda \neq 0$. In fact, if $M$ is a small sphere in $S^{3}$ of the form $M=\left\{\left(x^{1}, x^{2}, x^{3}, x^{4}\right) ; x^{4}=1 / \sqrt{2}\right\}$ then the norm of its mean curvature vector field is just 2. Therefore $\exp \Lambda \rho>f_{2}(\rho)$ for $\rho$ sufficiently near $\pi / 2$.

\title{
REFERENCES
}

[1] S.S. Chern, Minimal submanifolds in a Riemannian manifold, Mimeographed Lecture Notes, Univ. of Kansas (1968).

[2] D. Hoffman And J. Spruck, Sobolev and isoperimetric inequalities for Riemannian manifolds, Comm. Pure and Appl. Math., 27 (1974), 715-727.

[3] D. Hoffman and J. Spruck, A correction to [2], Comm. Pure and Appl. Math., 28 (1975), 765-766.

[4] S. Kobayashi and K. Nomizu, Foundations of differential geometry, Interscience, New York, vol. 2 (1969).

[5] J.H. Michael AND L.M. Simon, Sobolev and mean-value inequalities of generalized submanifolds of $R^{n}$, Comm. Pure and Appl. Math., 26 (1973), 361-379.

[6] R. Osserman, Isoperimetric and related inequalities, Proc. Symp. in Pure Math., 27 (1975), 207-215.

[7] T. ŌTsuki, Minimal hypersurfaces in a Riemannian manifold of constant curvature, Amer. J. Math., 92 (1970), 145-173.

\author{
Department of Mathematics \\ FACULTY OF EDUCATION \\ Tóyama University \\ TOYAMA, JAPAN
}

